\title{
A Prospective Observational Study on Treatment Evaluation in Patients Newly Diagnosed with Squamous Cell Carcinoma of Head and Neck in Bangladesh
}

\author{
*MA Hai ${ }^{1}, \mathrm{E} \mathrm{Haque}^{2}, \mathrm{~S} \mathrm{Reza}^{3}, \mathrm{M} \mathrm{Uddin}^{4}, \mathrm{~K}$ Pervin $^{5}$
}

\begin{abstract}
Background: Most head and neck cancers are squamous cell carcinomas (SCCHN) that contribute to substantial morbidity and mortality worldwide. The disease is mostly diagnosed at locally advanced stage. Because treatment of head and neck cancers is complex and involves multiple modalities of surgery, radiotherapy and systemic therapy including chemotherapy and molecularly targeted agents; a multidisciplinary approach is needed. The study evaluated the current treatment approaches for patients newly diagnosed with SCCHN in Bangladesh.
\end{abstract}

Methods: This prospective, observational and non-comparative, study enrolled eligible males and females of $\geqslant 18$ years newly diagnosed with SCCHN at any stage of disease. No therapeutic intervention was implied rather selection of treatment strategy and dosage of therapy was on discretion of individual oncologist as per routine clinical practice.

Results: A total of 64 patients with median age of 55 years were enrolled between August 2008 and July 2011. The majority of patients $(42,65.6 \%)$ were males. The major risk factors were betel leaf chewing along with jorda (smokeless tobacco) (62.5\%) and cigarette smoking (32.8\%), though most of the patients had multiple risk factors. No patient was diagnosed at early stage; all were in locally advanced disease at stage III $(52,81.3 \%)$ and IV (12, $18.8 \%)$. The oncologists prescribed neoadjuvant chemotherapy in half (32) of the patients, 19 (29.7\%) patients received adjuvant chemotherapy and $13(20.3 \%)$ received palliative chemotherapy. Only $3(4.6 \%)$ of the patients received radiotherapy. Chemotherapy combination regimen included docetaxel, cisplatin and 5-fluorouracil (5-FU) and leucovorin was added to 2 patients. The dosage of chemotherapeutic agents was as per routine clinical practice of the oncologists. Neutropenia was the common hematological abnormality reported spontaneously in 16 (25\%) patients. No serious adverse event was reported leading the patients to withdraw from therapy. During continuation of therapy 1 patient died due to sudden cardiac arrest who had medical history of previous myocardial infarction.

Conclusion: This registry revealed that squamous cell carcinoma of head and neck region are mostly presented in advanced stage in Bangladesh and the majority of the patients are treated with combination chemotherapeutic regimens.

Key Words: squamous cell carcinomas, head, neck, chemotherapy.

\section{Introduction}

Head and neck cancer encompasses epithelial malignancies that arise in the paranasal sinuses, nasal cavity, oral cavity, pharynx, and larynx. Almost all of the epithelial malignancies are squamous cell carcinoma of head and neck $(\mathrm{SCCHN})^{1,2}$ region and remains an important cause

of morbidity and mortality. ${ }^{3}$ Worldwide head and neck cancer is the sixth most common type of cancer, representing about $6 \%$ of all cases and accounting for an estimated annual burden of 650 000 new cancer cases and 350000 cancer deaths. ${ }^{4}$ Approximately $60 \%$ of the SCCHN are locally

$1^{*}$ Prof. Dr. MA Hai, Bangladesh Cancer Hospital and Welfare Home

${ }^{2}$ Prof. Dr. Ehteshamul Haque, Anwer Khan Modern Hospital Ltd.

${ }^{3}$ Dr. Salim Reza, Square Hospitals Ltd.

${ }^{4}$ Dr. Mokhles Uddin, Chittagong Medical College \& Hospital

${ }^{5}$ Dr. Kumkum Pervin, Sanofi Bangladesh

*Corresponding Author

Date of submission: 05.11.2017 Date of acceptance: 21.05.2018

AKMMC J 2019; 10(1) : 17-22 
advanced (stages III-IV) at presentation. ${ }^{5}$ Surgery and/or radiotherapy are the mainstay of locoregional treatment approaches and used as single modality therapy in early stage (I-II) of disease. ${ }^{6}$ For many years, radiotherapy has been the treatment of choice for locally advanced disease. ${ }^{7}$

The role of chemotherapy in SCCHN treatment has evolved from palliative care to a central component of curative programs for locally advanced SCCHN. ${ }^{8}$ A major advancement in the treatment of this stage of disease has been the introduction of concurrent administration of chemo-radiotherapy that has largely replaced radiotherapy alone in its treatment. ${ }^{7,9}$ Meta-analysis also suggests that addition of chemotherapy to loco-regional treatment specially in the form of concomitant/alternating chemo-radiotherapy results in absolute survival benefit in patients with SCCHN.10,11 Induction chemotherapy with docetaxel plus cisplatin and flurouracil has also been associated with improved survival benefit and could be a valuable treatment option. ${ }^{12}$ Improvement in surgical techniques and radiotherapy delivery and, moreover, incorporation of systemic therapy including chemotherapy and molecularly targeted agents (the epidermal growth factor receptor inhibitors) has been successfully integrated into potentially curative treatment with improved clinical outcomes. ${ }^{13}$ Treatment decisions in SCCHN are often complicated and collaboration of many specialties including head and neck surgeons, medical oncologists, radiation oncologists, radiologists, plastic surgeons, and dentists; is the key for optimum assessment and decision making.

Bangladesh is one of the Southeast Asian countries with incidence peaks of SCCHN. ${ }^{14}$ There is a lack of multidisciplinary approaches of disease management, hence, treatment strategy usually depends on physician's individual assessment and decision. However, still there is no consensus regarding standard therapy and reference regimens vary in treatment of SCCHN. We conducted this prospective survey on patients newly diagnosed with SCCHC at any stage of the disease to understand current treatment practice in Bangladesh to further improve care of these patients.

\section{Materials and Methods}

This was a prospective, observational, noncomparative, non-interventional study on treatment evaluation in patients newly diagnosed with SCCHN at any stage of the disease. Eligible patients were male or female aged 18 years or above having SCCHN that had been confirmed by histologic or cytologic analysis and diagnosed newly at any stage of the disease. A total of 50 patients was initially planned to be recruited by 8 oncologists (investigators) at their daily practice in Bangladesh. Patients presenting with concurrent history of other neoplasm were excluded for enrolment. Being noninterventional study, selection of treatment strategy and dosage of therapy were on discretion of individual oncologist as per routine clinical practice.

The investigators recorded patient data mainly on demographics, inclusion criteria, clinical stage and pathological grades of cancer, therapy plan at baseline. The actual therapy received was collected at the end of planned therapy received. All data was recorded in paper copy of Data Collection Forms (DCF) and the completed DCFs were collected by the study monitor at the end of study completion.

Statistical analysis was mainly descriptive and was summarized as mean, median, standard deviation, minimum, maximum and percentages. Statistical analysis was done using SPSS 17.0. The number of patients was determined from the estimated proportion of patients with head \& neck cancer treated by the physicians. To get optimum number of patients and considering the non-responders, drop out and design effect 50 patients were planned initially to include in this registry.

\section{Results}

A total of 64 patients were enrolled between August 2008 and July 2011, 42 (65.6\%) were males and 22 $(34.4 \%)$ were females. The median age of the patients was 55 years with the youngest one at 26 years and the oldest one at 101 years and $67.2 \%$ (43) were between 41 to 60 years. The major risk factors identified for the SCCHN were betel leaf chewing along with jorda (smokeless tobacco) $(62.5 \%)$, cigarette smoking $(32.8 \%)$ and family history of cancer $(6.3 \%)$. Most of the patients had multiple risk factors. Half (21) of the male patients 
were smokers and $57.1 \%$ (24) had habit of chewing betel leaf along with jorda. Though female patients had no history of active smoking but about threefourth $(72.7 \%)$ of them were chewing betel leaf along with jorda. The common comorbidities associated were hypertension (43.8\%), anemia $(34.4 \%)$, diabetes $(29.7 \%)$, chronic upper respiratory tract infections $(6.3 \%)$, and myocardial infarction $(4.7 \%)$. The demographic and clinical characteristics of study patients are presented in Table 1.

Table 1: Baseline demographics and clinical characteristics of study patients

\begin{tabular}{|c|c|c|c|}
\hline Demographics (mean \pm SD) & $\begin{array}{c}\text { Male } \\
(n=42)\end{array}$ & $\begin{array}{l}\text { Female } \\
(\mathrm{n}=22)\end{array}$ & $\begin{array}{c}\text { Total } \\
(n=64)\end{array}$ \\
\hline Age (yrs) & $57.3 \pm 14.2$ & $51.3 \pm 9.0$ & $55.2 \pm 12.9$ \\
\hline Height (cm) & $160.9 \pm 5.8$ & $149.6 \pm 7.1$ & $157.0 \pm 8.2$ \\
\hline Weight (kg) & $60.5 \pm 8.01$ & $51.5 \pm 6.0$ & $57.4 \pm 8.4$ \\
\hline $\operatorname{BMI}\left(\mathrm{kg} / \mathrm{m}^{2}\right)$ & $23.3 \pm 2.5$ & $23.0 \pm 2.3$ & $23.2 \pm 2.4$ \\
\hline \multicolumn{4}{|l|}{$\begin{array}{l}\text { Risk factors and main medical } \\
\text { history }(n, \%)\end{array}$} \\
\hline History of major illness & $3(7.1)$ & 0 & $3(4.7)$ \\
\hline Family history of cancer & $3(7.1)$ & $1(4.5)$ & $4(6.3)$ \\
\hline Smoker (current and previous) & $21(50)$ & 0 & $21(32.8)$ \\
\hline Betel leaf chewing along with jorda & $24(57.1)$ & $16(72.7)$ & $40(62.5)$ \\
\hline Diabetes & $8(19)$ & $11(50)$ & $19(29.7)$ \\
\hline Hypertension & $14(33.3)$ & $14(63.6)$ & $28(43.8)$ \\
\hline Anemia & $12(28.6)$ & $10(45.5)$ & $22(34.4)$ \\
\hline Chronic upper respiratory tract infection & n $4(9.5)$ & 0 & $4(6.3)$ \\
\hline Myocardial infraction & $3(7.1)$ & 0 & $3(4.7)$ \\
\hline \multicolumn{4}{|l|}{$\begin{array}{l}\text { Signs and Symptoms } \\
(\mathrm{n}, \%)\end{array}$} \\
\hline Sore throat & $29(69.0)$ & $12(54.5)$ & $41(64.1)$ \\
\hline Oral ulceration & $19(45.2)$ & $13(59.1)$ & $32(50.0)$ \\
\hline Dysphagia & $27(64.3)$ & $13(59.1)$ & $40(62.5)$ \\
\hline Otalgia: Local/referred & $6(14.3)$ & $7(31.8)$ & $13(20.3)$ \\
\hline Haemoptysis & $6(14.3)$ & $2(9.1)$ & $8(12.5)$ \\
\hline Persistent cough & $1638.1)$ & $3(13.6)$ & $19(29.7)$ \\
\hline Epistaxis & $3(7.1)$ & $2(9.1)$ & $5(7.8)$ \\
\hline Serous Otitis Media & $1(2.4)$ & 0 & $1(1.6)$ \\
\hline Anorexia & $30(71.4)$ & $11(50.0)$ & $41(64.1)$ \\
\hline Fatigue & $34(81.0)$ & $14(63.6)$ & $48(75.0)$ \\
\hline Weight loss & $31(73.8)$ & $16(72.7)$ & $47(73.4)$ \\
\hline Hoarseness of voice & $21(50.0)$ & $8(46.4)$ & $29(45.3)$ \\
\hline Loss of appetite & $26(61.9)$ & $14(63.6)$ & $40(62.5)$ \\
\hline Hearing loss & $3(7.1)$ & $1(4.5)$ & $4(6.3)$ \\
\hline Local swelling & $20(47.6)$ & $3(13.6)$ & $23(35.9)$ \\
\hline
\end{tabular}

Fatigue $(75 \%)$, weight loss $(73.4 \%)$, sore throat $(64.1 \%)$, anorexia $(64.1 \%)$, loss of appetite (62.5\%), and dysphagia (62.5\%) were the common sign/symptoms reported by the patients. In $50 \%$ of the patients oral ulceration was found on examination. At inclusion 14 (21.9\%) patients had ECOG score-1, 42 (65.6\%) had ECOG score-2 and $8(12 \%)$ patients had ECOG score-3.

For evaluation of the tumor malignancy the methods used by the investigators were inspection of mucosa $(65.6 \%)$, palpation of neck $(61 \%)$, endoscopic examination (direct laryngoscopy, esophagoscopy) (45.3\%) and bimanual examination of oral cavity $(32.8 \%)$. Indirect laryngoscopy was done for $10.9 \%$ of the cases. On evaluation 27 $(42.2 \%)$ patients had positive lesions in pharynx and laryngeal area, $15(23.4 \%)$ had in oral cavity and $13(20.3 \%)$ in tongue. The lesions were categorized as residual growth (25), oedematous (11) and congested erosion (5). Neck nodes were positive in $9(14.4 \%)$ patients.

Fine needle aspiration cytology (FNAC) was done for cytological examination. Histologically more than half of the tumors $(53.1 \%)$ were moderately differentiated and $12.5 \%$ were well differentiated. However, $28.1 \%$ of those were poorly differentiated and $6.3 \%$ were undifferentiated. The median tumor size was $4 \mathrm{~cm}$ with the smallest measurement at $0.5 \mathrm{~cm}$ and largest at $8.5 \mathrm{~cm}$. Tumor size was measured by physical examination or CT scan or both. The tumor among male patients were little larger than that of females. The patients were presented with locally advanced cancers and $81.3 \%$ (52) had stage III and $18.8 \%$ (12) had stage IV disease at the diagnosis. TNM staging of the disease is presented in Table 2. 
Table 2: Stage of the disease in patients

\begin{tabular}{ccc}
\hline & $\begin{array}{c}\text { Frequency } \\
(\mathbf{n}=\mathbf{6 4})\end{array}$ & $\begin{array}{c}\text { Percent } \\
\mathbf{( \% )}\end{array}$ \\
\hline Stage of tumor & 9 & 14.1 \\
T1 & 27 & 42.2 \\
T2 & 16 & 25.0 \\
T3 & 12 & 18.8 \\
T4 & & \\
N0 & 8 & 12.5 \\
N1 & 43 & 67.2 \\
N2 & 11 & 17.2 \\
N3 & 2 & 3.1 \\
M0 & & \\
M1 & 59 & 92.2 \\
Metastasis Status & 5 & 7.8 \\
Overall stage of disease & & 81.3 \\
Stage III & 52 & 18.8 \\
Stage IV & 12 & \\
\hline
\end{tabular}

For treating the SCCHN the investigators planned chemotherapy for 62 patients including neoadjuvant chemotherapy for 31 (48\%) patients, adjuvant chemotherapy for $18(28.1 \%)$ patients and palliative

Table 3: Plan and actual therapy received
Docetaxel $\left(\right.$ Taxotere $\left.^{\circledR}\right)$, cisplatin and 5-fluorouracil (5-FU) were the regimen prescribed to $96 \%$ (62) of the patients. Only 3\% (02) of the patients were prescribed leucovorin in addition to that regimen. The dosage of docetaxel, cisplatin and 5-FU were decided by the physicians as per routine clinical practice. The median dose of docetaxel was $80 \mathrm{mg}$, the dose of cisplatin was $100 \mathrm{mg}$ and that of 5-FU was $900 \mathrm{mg}$.

At the end of therapy there was an improvement of performance status in $39(60.9 \%)$ patients having ECOG score-2 and 17 (26.6\%) having ECOG score-1 at inclusion visit. However, the changes were not statistically significant $(p=0.284)$. There was no change in patients $(8)$ having ECOG score-3.

Neutropenia was the common hematological abnormality reported spontaneously in $16(25 \%)$ patients. No serious adverse event was reported leading the patients to withdraw from therapy. During continuation of therapy 1 patient died due to sudden cardiac arrest who had medical history of previous myocardial infarction.

\begin{tabular}{lccccccc}
\hline & $\begin{array}{c}\text { Therapy plan } \\
(\mathbf{n}=\mathbf{6 4})\end{array}$ & \multicolumn{3}{c}{$\begin{array}{c}\text { Before treatment } \\
\mathbf{n}(\boldsymbol{\%})\end{array}$} & \multicolumn{3}{c}{$\begin{array}{c}\text { Actual therapy received } \\
\mathbf{n}(\%)\end{array}$} \\
\hline & & Male & Female & Total & Male & Female & Total \\
& & $\mathbf{N}=\mathbf{4 2}$ & $\mathbf{N = 2 2}$ & $\mathbf{N = 6 4}$ & $\mathbf{N}=\mathbf{4 2}$ & $\mathbf{N = \mathbf { 2 2 }}$ & $\mathbf{N = 6 4}$ \\
\hline Chemotherapy & Neoadjuvant & $24(57.1)$ & $7(31.8)$ & $31(48.4)$ & $25(59.5)$ & $7(31.8)$ & $32(50)$ \\
& Adjuvant & $7(16.7)$ & $11(50.0)$ & $18(28.1)$ & $7(16.7)$ & $12(54.5)$ & $19(29.7)$ \\
& Palliative / & $9(21.4)$ & $4(18.2)$ & $13(20.3)$ & $10(23.8)$ & $3(13.6)$ & $13(20.3)$ \\
& Non Curative & & & & & & \\
Radiotherapy & Neo adjuvant & $2(4.8)$ & 0 & $2(3.1)$ & $3(7.14)$ & 0 & $3(4.6)$
\end{tabular}

chemotherapy for 13 (20.3\%) patients. At the end of the therapy it was found that $61(95.3 \%)$ patients received chemotherapy and $3(4.6 \%)$ received radiotherapy. Neoadjuvant chemotherapy was received by $32(50 \%)$ patients, $19(29.7 \%)$ received adjuvant chemotherapy and $13(20.3 \%)$ received palliative chemotherapy as planned before treatment. Table 3 shows details of planned and actual therapy received by the patients.

\section{Discussion}

In this study the patients with SCCHN were presented in locally advanced disease with males affected more frequently at early at the age of 26 years and even at 101 years. The major risk factors identified were betel leaf chewing along with jorda (smokeless tobacco) and cigarette smoking and family history of cancer was another 
factor contributing to the disease. Though female patients had no history of active smoking but about three-fourth of them were chewing betel leaf along with jorda. The patients presented with common sign-symptoms of fatigue, weight loss, sore throat, anorexia/loss of appetite and dysphagia. Positive lesions were present in pharynx, laryngeal area, oral cavity and tongue. Neck nodes were also positive in some of the patients. The role of chemotherapy in SCCHN treatment has evolved from palliative care to a central component of curative programs for locally advanced SCCHN. ${ }^{8}$ A major advancement in the treatment of this stage of disease has been the introduction of concurrent administration of chemoradiotherapy that has largely replaced radiotherapy alone in its treatment. ${ }^{7,9}$ Metaanalysis also suggests that addition of chemotherapy to locoregional treatment specially in the form of concomitant/alternating chemoradiotherapy results in absolute survival benefit in patients with SCCHN. ${ }^{10,11}$ Induction chemotherapy with docetaxel plus cisplatin and flurouracil has also been associated with improved survival benefit and could be a valuable treatment option. ${ }^{12}$ Improvement in surgical techniques and radiotherapy delivery and, moreover, incorporation of systemic therapy including chemotherapy and molecularly targeted agents (the epidermal growth factor receptor inhibitors) has been successfully integrated into potentially curative treatment with improved clinical outcomes. ${ }^{13}$

On evaluation at the end of therapy it was found that all most all $(95.3 \%)$ of the patients received chemotherapy while very few $(4.6 \%)$ patients received radiotherapy for treatment of SSHNC. Chemotherapy included neoadjuvant therapy in 32 patients, adjuvant therapy in 19 patients and palliative chemotherapy in 13 patients. Docetaxel, cisplatin and 5-fluorouracil (5-FU) were the regimen prescribed to the patients. Leucovorin was also prescribed in addition to that regimen though the frequency was very low. Neutropenia was the common hematological abnormality reported spontaneously in $25 \%$ of patients. No serious adverse event was reported leading the patient to withdraw from therapy. One patient died during continuation of therapy due to sudden cardiac arrest who had medical history of previous myocardial infarction.

\section{Conclusion}

This registry revealed that squamous cell carcinoma of head and neck region are mostly presented in advanced stage in Bangladesh and the majority of the patients are treated with combination chemotherapeutic regimens. As multidisciplinary treatment approach and referral system is not established in Bangladesh decision about treatment strategy depends largely on treating physician's own discretion. However, in deciding treatment strategy suitable for individual patient, important considerations should include tumor site, stage and resectability, desire for organ preservation, expected functional outcomes, ability to tolerate treatment, and comorbid illnesses.

\section{Acknowledgement}

The study was supported by Sanofi Bangladesh Limited. The authors who were investigators of this study and received investigator fees from Sanofi Bangladesh for conducting the study.

\section{Conflict of interest: none.}

\section{References}

1. American Cancer Society. Cancer Facts \& Figures 2013. Atlanta, Ga: American Cancer Society; 2013.

2. American Joint Committee on Cancer. Lip and Oral Cavity. In: AJCC Cancer Staging Manual, 7th ed. New York, Springer: 2010; 29-35.

3. Ferlay J, Shin HR, Bray F, et al. Estimates of worldwide burden of cancer in 2008: GLOBOCAN 2008. Int J Cancer 2010; 127: 2893-2917.

4. Parkin DM, Bray F, Ferlay J, et al. Global cancer statistics, 2002. CA Cancer J Clin 2005; 55: $74-108$

5. Seiwert TY, Cohen EEW. State-of-the art management of locally advanced head and neck cancer. Br J Cancer 2005; 92: 1341-8]. 
6. Vokes EE, Weichselbaum RR, Lippman SM, et al. Head and neck cancer. N Engl J Med 1993; 328: 184-94.]

7. Lefebvre J-L. Current clinical outcomes demand new treatment options for SCCHN. Ann Oncol 2005; 16: Suppl 6: vi7-vi12.]

8. Cohen EE, Lingen MW, Vokes EE. The expanding role of systemic therapy in head and neck cancer. J Clin Oncol 2004; 22: 1743-52.

9. Adelstein DJ, Li Y, Adams G, et al. An intergroup phase III comparison of standard radiation therapy and two schedules of concurrent chemoradiotherapy in patients with unresectable squamous cell head and neck cancer. J Clin Oncol 2003; 21: 92-8.

10. Pignon JP, Bourhis J, Domenge C, et al. on behalf of the MACH-NC Collaborative Group. Chemotherapy added to locoregional treatment for head and neck squamous-cell carcinoma: three meta-analyses of updated individual data. Lancet 2000; 355: 949-55.
11. Bourhis J, Amand C, Pignon JP. Update of MACH-NC (Meta-Analysis of Chemotherapy in Head \& Neck Cancer) database focused on concomitant chemoradiotherapy. J Clin Oncol 2004; 22: 5505.

12. Jan B. Vermorken, Eva Remenar, Carla van Herpen, et al. Cisplatin, fluorouracil, and docetaxel in unresectable head and neck cancer. N Engl J Med 2007; 357: 1695-704.

13. A Argiris; M V Karamouzis; R L Ferris; D Raben. Head and neck cancer. Lancet 2008; 371: 1695-709

14. Ferlay J, Shin HR, Bray F, Forman D, Mathers C, Parkin DM. GLOBOCAN 2008, Cancer Incidence and Mortality Worldwide: IARC Cancer Base No. 10 Lyon, France: International Agency for Research on Cancer; 2010. Available from: http://globocan.iarc.fr. 